\title{
Carbon nanotube yarns: Sensors, actuators and current carriers
}

\author{
Tissaphern Mirfakhrai ${ }^{*}$, Mikhail Kozlov ${ }^{\mathrm{b}}$, Shaoli Fang ${ }^{\mathrm{b}}$, Mei Zhang ${ }^{\mathrm{b}}$, \\ Ray H. Baughman ${ }^{\mathrm{b}}$ and John D Madden ${ }^{\mathrm{a}}$ \\ ${ }^{\mathrm{a}}$ Dept. of Electrical and Computer Engineering and Advanced Materials and Process Engineering \\ Laboratory, University of British Columbia, Vancouver BC, V6T 1Z4, Canada; \\ ${ }^{b}$ Alan G. MacDiarmid NanoTech Institute, University of Texas at Dallas, Richardson, TX 75080, \\ USA
}

\begin{abstract}
Carbon nanotubes (CNTs) have attracted extensive attention in the past few years because of their appealing mechanical and electronic properties. Yarns made through spinning multi-walled carbon nanotubes (MWNTs) have been reported. Here we report the application of these yarns as electrochemical actuators, force sensors and microwires. When extra charge is stored in the yarns, change in length. This actuation is thought to be because of electrostatic as well as quantum chemical effects in the nanotube backbones. We report strains up to $0.7 \%$. At the same time, the charged yarns can respond to a change in the applied tension by generating a current or a potential difference that is related to the applied tension force. As current carriers, the yarns offer a conductivity of $\sim 300 \mathrm{~S} / \mathrm{cm}$, which increases linearly with temperature. We report a current capacity of more than $10^{8} \mathrm{~A} / \mathrm{m}^{2}$, which is comparable to those of macroscopic metal wires. However, these nanotube yarns have a density $\left(0.8 \mathrm{~g} / \mathrm{cm}^{3}\right)$ that is an order of magnitude lower than metallic wires. The MWNT yarns are mechanically strong with tensile strengths reaching $700 \mathrm{MPa}$. These properties together make them a candidate material for use in many applications including sensors, actuators and light-weight current carriers
\end{abstract}

Keywords: Carbon Nanotubes, actuators, sensors, current carriers

\section{INTRODUCTION}

Carbon nanotubes (CNTs) have been shown to have high modulus $(0.7 \mathrm{TPa})$, and high strength (30 GPa), while also displaying high electrical $(10-30 \mathrm{kS} / \mathrm{cm})$ and thermal $(2000 \mathrm{~W} / \mathrm{mK})$ conductivities [1]. The low density of CNTs makes the specific properties even more useful, hence the interest of technologists in developing applications. A difficulty delaying commercial applications has been the absence of methods for controlling the trillions of CNTs as they are being assembled into macro-sized objects. The main methods of assembling fibres have been to disperse the CNTs into fluids and either extrude or coagulation-spin the dispersions into fibres. More crudely the CNTs in these dispersions can also be filtered out of solution to make papers. A recently demonstrated alternative to these solution-based methods is to create twist-spun yarns of multi-walled carbon nanotubes by drawing from aligned forests of nanotubes (Figure 1a) [2].The MWNTs in these yarns are believed to be held together by strong van der Waals forces that arise due to the interactions on the long and smooth surfaces of the MWNTs. The yarns can be plied together to form multi-ply yarns, capable of maintaining even higher tensions (Figure 1b). These yarns offer exceptional mechanical properties such as very high gravimetric modulus and tensile strengths of about $750 \mathrm{MPa}$ [3]. It has been shown that a dimensional change takes place in the yarns when a voltage is applied to them in an electrolyte [4]. Due to the very high tensile strength and Young's modulus of the yarns, the yarns can operate at high forces and produce unprecedented work densities.

Piezoelectrics, electric motors, conducting polymers [5] and many other actuators can be operated as sensors or generators as well as actuators. We report that if a force is applied to a charged yarn in an electrolyte, it also can generate a change in its potential or short-circuit current. Therefore, the yarn can be used as a force sensor. Also, CNT yarns can carry a lot of current, with the current carrying capacity being particularly impressive when normalized by density. At about $300 \mathrm{~S} / \mathrm{cm}$, the conductivity of these yarns exceeds that of most organic conductors. Their high tensile strength, along with their large current capacity and high density-normalized conductivity makes them potential candidate as current carriers and interconnects. Because their density is quite low $\left(0.8 \mathrm{~g} / \mathrm{cm}^{3}\right)$ they are especially suited to be used as

\footnotetext{
*Corresponding author: tissa@ece.ubc.ca
}

Electroactive Polymer Actuators and Devices (EAPAD) 2008, edited by Yoseph Bar-Cohen Proc. of SPIE Vol. 6927, 692708, (2008) · 0277-786X/08/\$18 · doi: 10.1117/12.775476 
current carriers in aerospace applications where the weight of the equipment is a major concern. In following sections, we discuss the application of these MWNT yarns as actuators, sensors and current carriers, preceded by a description of the experimental methods.

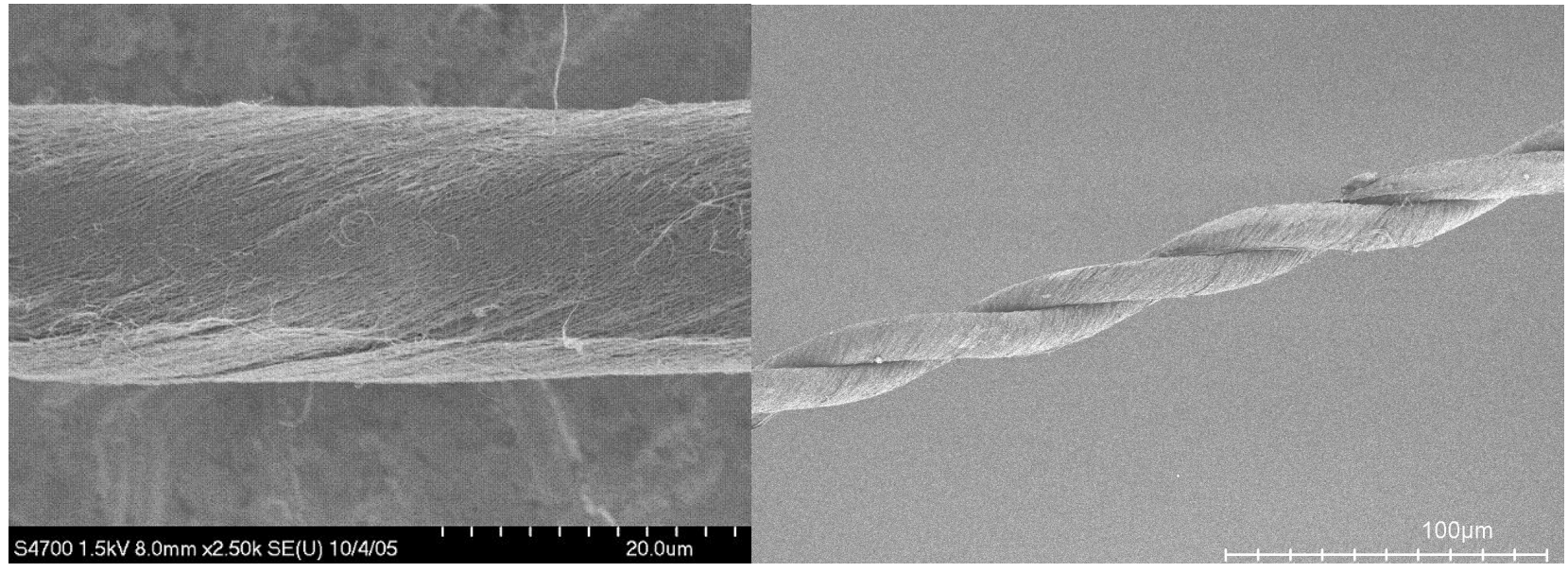

(a)

(b)

Figure 1: Scanning electron micrographs of (a) a single-ply yarn and (b) a two-ply yarn spun using the dry twist-spinning technique.

\section{METHODS}

MWNT yarns were prepared by a dry spinning method [2], in which they are drawn from a forest and twisted into yarns. The MWNT forest was synthesized by catalytic CVD using acetylene gas as the carbon source. MWNTs, which are about $10 \mathrm{~nm}$ in diameter, are simultaneously drawn from the MWNT forest and twisted. The diameter of the samples used was about $10 \mu \mathrm{m}$. The twist is characterized by the helix angle $(\alpha)$, which depends directly upon the degree of twist and inversely on the yarn diameter. The degree of twist is typically 13500 turns $/ \mathrm{m}$, corresponding to a twist angle of about 23 degrees.

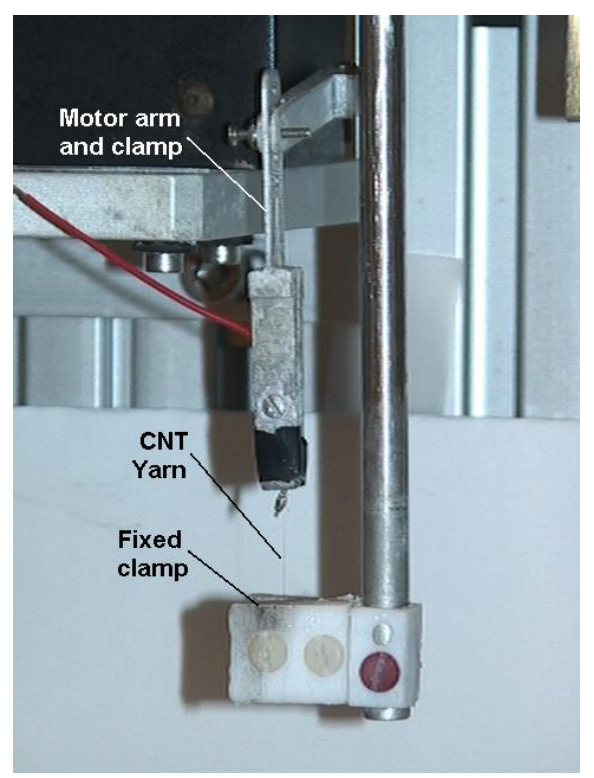

Figure 2: A CNT yarn mounted in the ASI muscle analyzer clamp.

In order to apply forces and record actuation forces and displacements generated by the CNT yarns, an Aurora Scientific ASI 300 muscle analyzer and its LabView-based software (www.aurorascientific.com) have been employed. 
Figure 2 is a photo of the apparatus used to apply force and of the yarn and clamping mechanism. The fiber is mounted between a lower fixed clamp and an upper movable motor arm. A computer- controlled potentiostat is used to apply potentials. A data acquisition card (National Instruments 6036E) gathers the force and displacement data from the muscle analyzer and also logs the applied voltage and current. Figure 3 is a drawing of the measurement setup with the yarn as one of the electrodes in the actuation cell. The ASI 300 instrument can be programmed to keep the load on the yarn constant and to measure the displacement generated by the yarn, or it can keep the length of the yarn constant and measure the tension change in the yarn due to actuation. Finally, it can also be used to apply a desired force profile as a function of time and measure the resulting changes in the length of the sample.

To study actuation, a voltage is applied using the potentiostat between the yarn, which is the working electrode (WE) and a piece of porous carbon fiber paper acting as the counter electrode (CE). The capacitance of the CE is much larger than that of the yarn. The potential between WE and an $\mathrm{Ag}+$ reference electrode (RE) is used as a measure of the potential across the WE, and this potential is controlled using a potentiostat. The potential between WE and CE is controlled such that the potential between WE and RE follows the desired values and profiles.

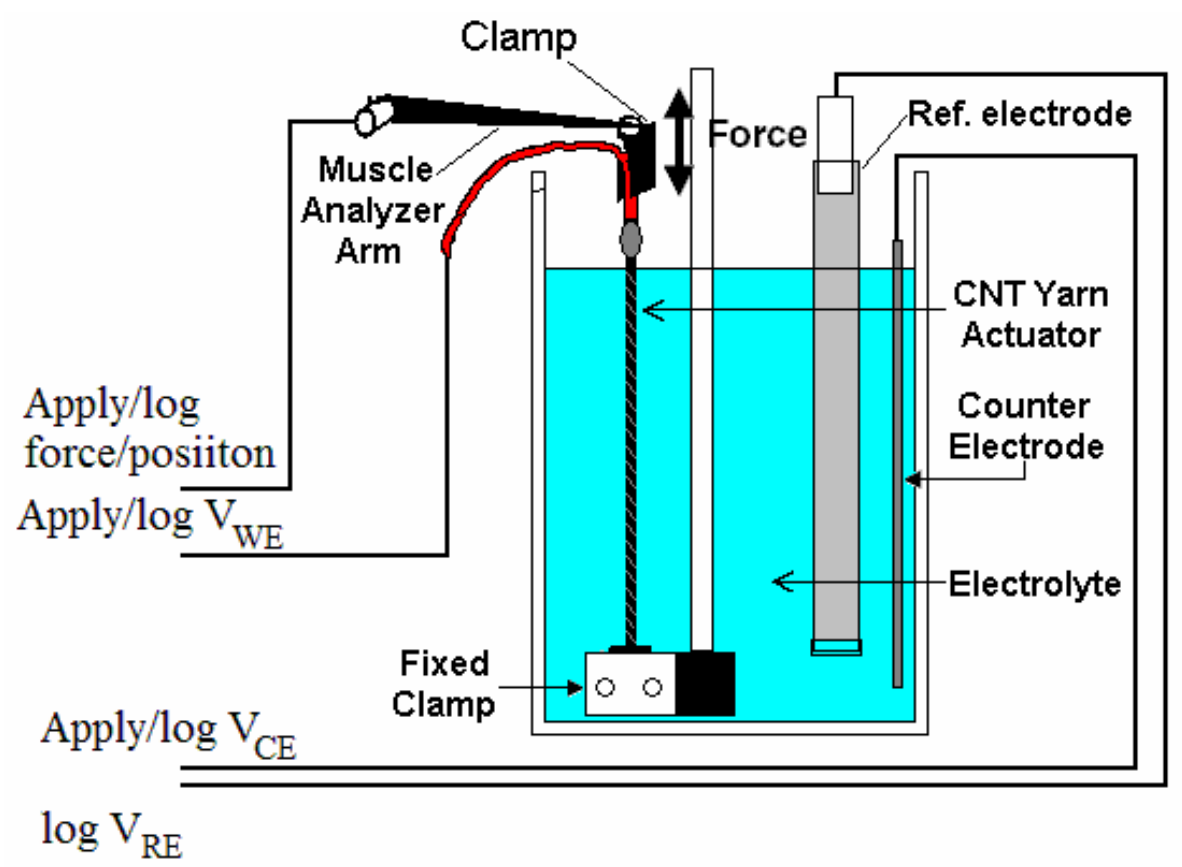

Figure 3: Electrochemical cell setup used for actuation and sensory tests.

\section{ACTUATORS}

It is observed that when a potential is applied and varied under constant load, the length of the yarn starts to change. Figure 4a shows the strain (adjusted for creep) generated in the yarn when the potential between the WE and RE is changed as shown in Figure $4 \mathrm{~b}$. The mechanism of this actuation, which is in the form of contraction upon the application of the voltage, is not completely understood. However, Coulombic interactions between the adjacent MWNTs as well as quantum mechanical effects within each MWNT may be at work [6]. 

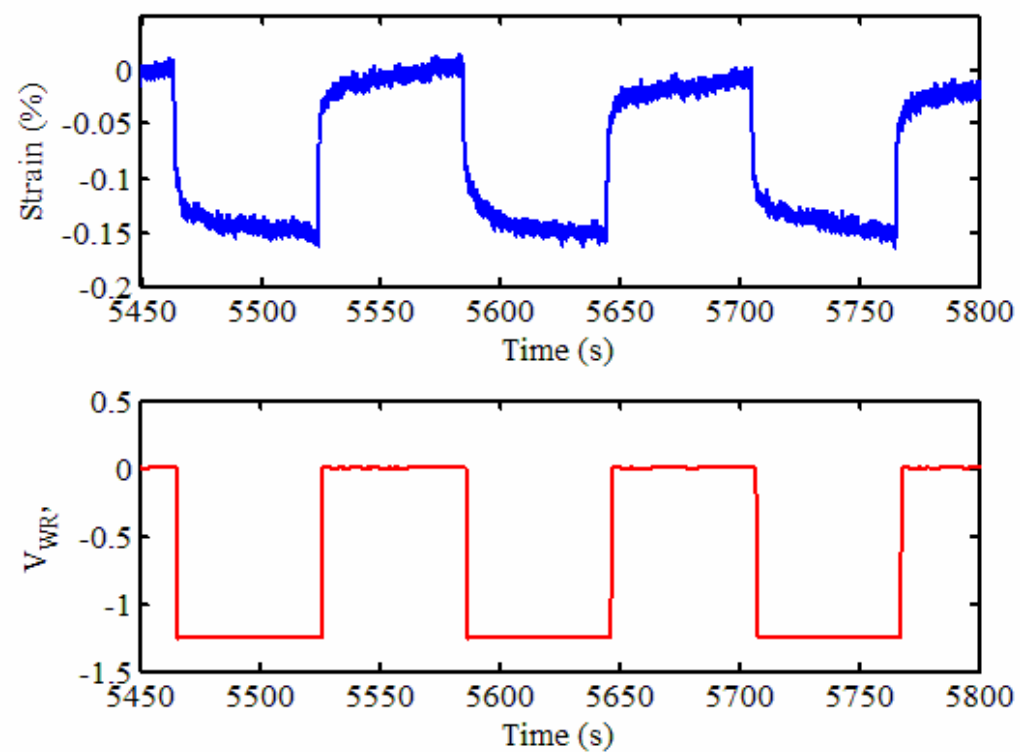

Figure 4: Actuation strain response to an applied square wave potential of $-1.25 \mathrm{~V}$ amplitude between the working electrode (yarn) and a $\mathrm{Ag} / \mathrm{Ag}+$ reference electrode in an electrolyte of $0.1 \mathrm{M}$ tetrabutyleammonium hexafluorophosphate (TBAP) in acetonitrile.

A series of voltage pulses with various amplitudes were applied to the yarn and the resulting actuation strains are plotted in Figure 5. Because the Young's modulus of the yarn is about $20 \mathrm{GPa}$, even though the strains are relatively small, they translate to stresses of more than $14 \mathrm{MPa}$. The tensile strength of the yarn is about $550 \mathrm{MPa}$, and we have previously shown that actuation strain does not diminish when the load is increased [4]. Strain value can vary in different electrolytes. In principle loads potentially as large as $550 \mathrm{MPa}$ can be displaced by as much as $0.7 \%$ by the yarn actuators, resulting in high coupling [7].

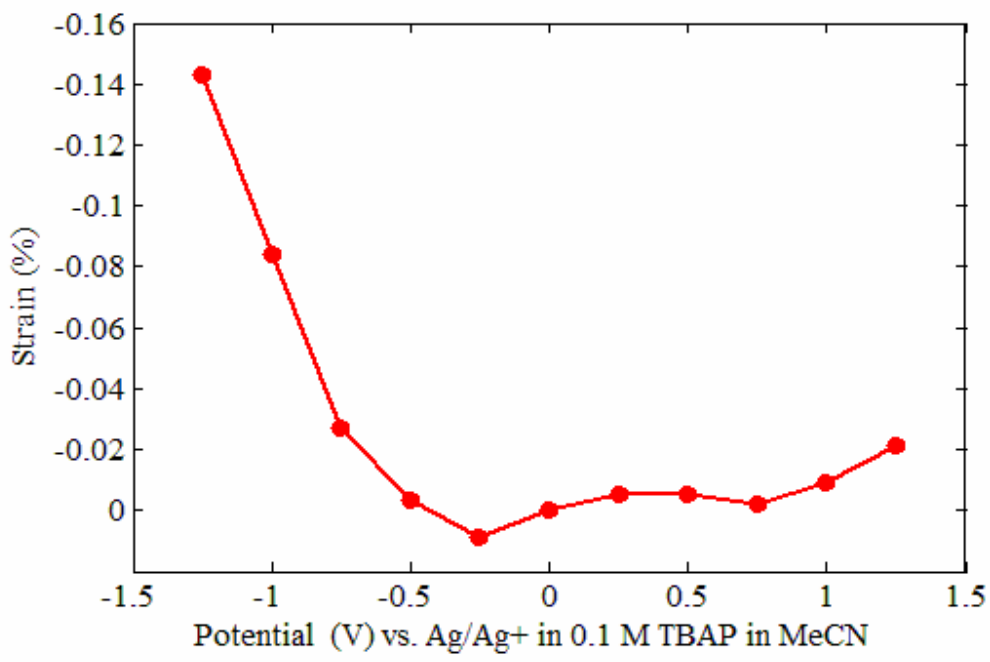

Figure 5: Actuation strain response to applied square wave potentials from $-1.25 \mathrm{~V}$ to $+1.25 \mathrm{~V}$ between the working electrode (yarn) and an $\mathrm{Ag} / \mathrm{Ag}+$ reference electrode in an electrolyte of $0.1 \mathrm{M}$ tetrabutylammonium hexafluorophosphate (TBAP) in acetonitrile. 


\section{FORCE SENSORS}

Many actuators can also act as generators and sensors if a force is applied to them. For example, an electric motor can also act as a generator. Here we report the results of investigating generation of electric current/voltage when a force is applied to a yarn. When a force is applied to the yarn biased to a given voltage in an electrolyte, a spike is observed in the current (Figure 6). Figure 7 shows the amplitude of these sensory current spikes plotted as a function of the applied bias potential between the WE and RE. The amplitude is plotted at three different levels of the amplitude of the stress pulse at 15,30, and $45 \mathrm{MPa}$, at a prestress of $30 \mathrm{MPa}$. The example in Figure 6 is at $+0.5 \mathrm{~V}$, while the stress is changed by $45 \mathrm{MPa}$.
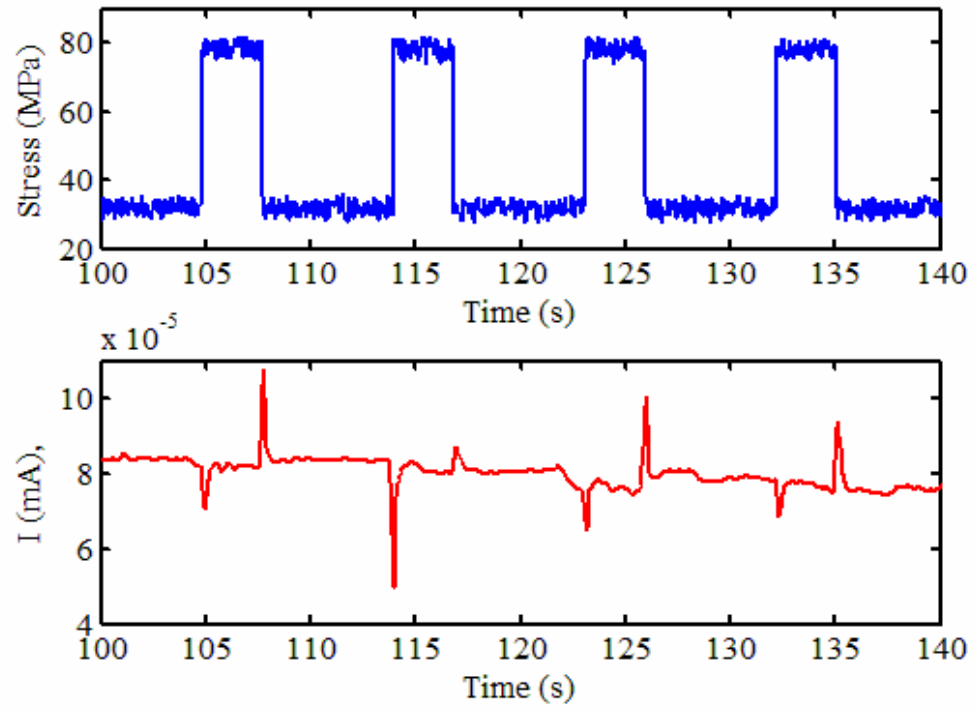

Figure 6: (a) Stress profile applied to the yarn under constant potential generates a train of current spikes (b)

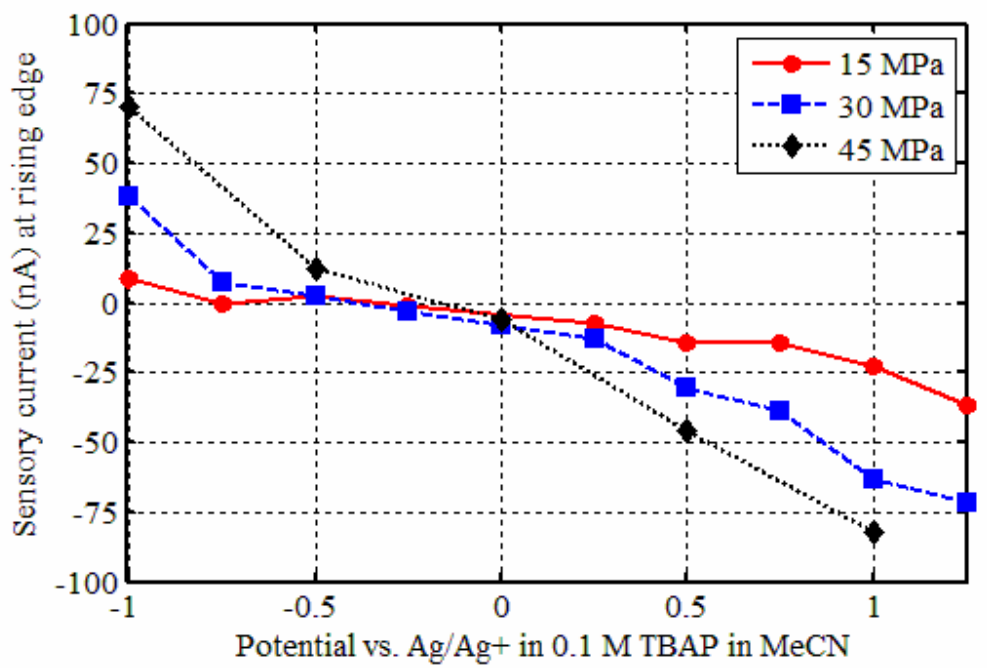

Figure 7: Sensory current as a function of the applied bias potential at three different loads.

One may be tempted to say that by pulling on the yarn, one is stretching the individual parallel CNTs in it, and thus varying the capacitance. However, considering that the Poisson's ratio of MWNTs is about 0.2 to 0.3 [8], applying 
strains in the range used in our experiments will result in an expansion of the surface area of the MWNTs. An increase in surface are of a conductor will typically increase its capacitance. If the yarn is biased to a positive potential, it includes extra negative ions from the electrolyte. Under such circumstances and based on the theory under consideration, if the yarns is pulled, its capacitance will increase and it will tend to pull in more negative ions from the electrolyte, resulting in a positive current spike. Since this prediction is contradicted by the experiments, we must assume that a different mechanism is at work.

One possibility is that ions are squeezed out of the nanotubes, producing a change due to charge expulsion, similar to the mechanism that has been proposed to explain similar sensor behaviour in conducting polymers. When a positive bias potential is applied to the yarn, negative ions are absorbed at the surface of the yarn due to Coulomb forces and gradually penetrate the yarn. When a force is applied to the yarn, the axial tension compacts the yarn radially [9]. This radial compaction reduces the inner surface area available for interaction with the ions, thus expelling the negative ions that have been inside. The eviction of the negative ions results in an inward current into the yarn. A current flowing into the yarn working electrode is negative (by convention). The current is reversed when a negative bias potential is applied to the yarn, resulting in a flux of negative ions into the yarn, and a positive current flowing outward from the yarn.

The fact that carbon nanotube yarns can act as both sensors and actuators is an exciting observation. More investigation is needed to understand their behaviour and a theory is being developed to quantitatively account for the sensory effects [10]. We have already demonstrated that the yarns can work as actuators at loads of up to $120 \mathrm{MPa}$ and we see no reason that they cannot operate as sensors up to at least the same load levels. If they can operate at close to their tensile strength of about $750 \mathrm{MPa}$, it will result in a mechanical stress sensor with unprecedented load levels. Since nanotubes are stable at temperatures of up to $450{ }^{\circ} \mathrm{C}$ in air and to more than $1000{ }^{\circ} \mathrm{C}$ in the absence of oxygen, applications as hightemperature force sensors can be contemplated. In such circumstances the electrolyte has to be a molten salt.

\section{CURRENT CARRIERS AND INTERCONNECTS}

To determine the effect of temperature on the conductivity a 4-point probe was used. The temperature was controlled using a hot plate. A potential difference of $0.1 \mathrm{~V}$ was applied to the yarn at all temperatures. The temperature was increased from room temperature to $90{ }^{\circ} \mathrm{C}$ and then back to room temperature. The conductivity is found to linearly increase with temperature with a slope of $0.33 \mathrm{~S} / \mathrm{cm}$ per ${ }^{\circ} \mathrm{C}$. The conductivity during warming and cooling is plotted in Figure 8 as a function of temperature. Since the yarn is made of numerous nanotubes, the increase in conductivity may be due to a phonon-assisted mechanism such as the one proposed for conductivity in other fullerenes [11] or could be due the fact contribution of semiconducting shells in the MWNTs to the total conductivity becomes comparable to metallic ones [12].

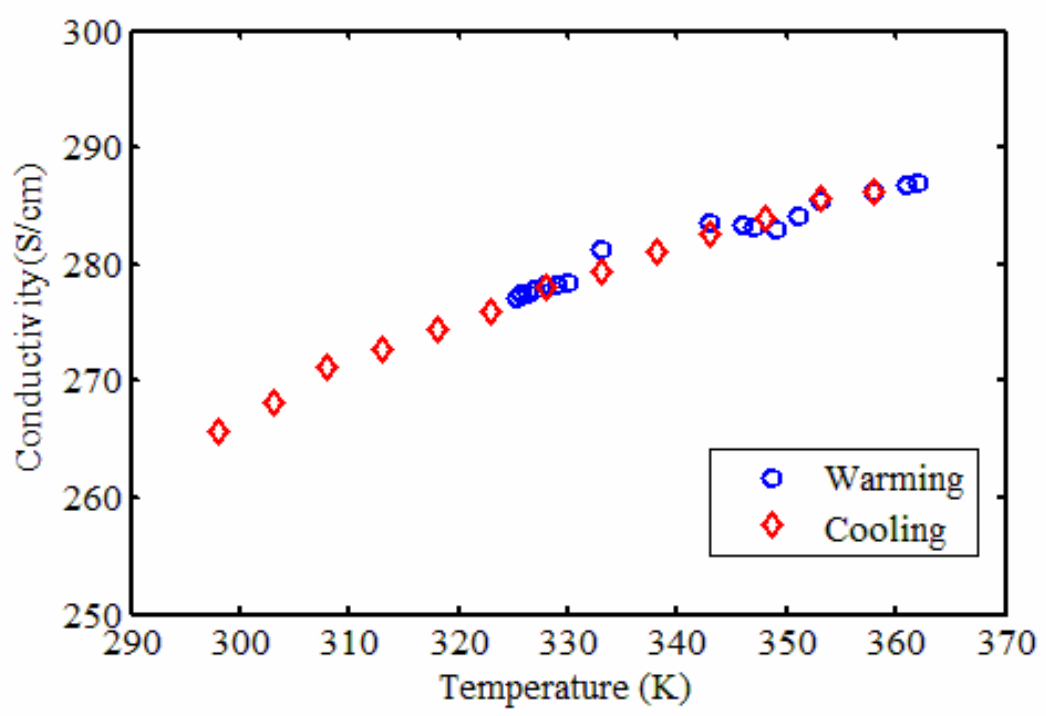

Figure 8: The conductivity of a single-ply yarn kept at constant temperature. The applied voltage is $0.1 \mathrm{~V}$. 
The current density of the yarn in air was also measured using a 4-point probe. The applied potential difference was increased and the current was measured until the yarn failed. The mechanism of this failure is not yet completely understood. A likely cause for the breakage of the yarn is the oxidization of the yarn when the current (applied voltage) is high. The break point is likely where the current density is highest. The fact that the yarn always physically breaks at the point where the current enters the yarn seems to confirm this. Figure 9 shows the current density as a function of the applied voltage. For comparison, the max current density allowed in most commercial copper wires is about $2 \times 10^{7} \frac{\mathrm{A}}{\mathrm{m}^{2}}$. The improvement in current carrying capacity over bulk copper is even more exciting when we consider that the density of copper is $8.92 \mathrm{~g} / \mathrm{cm}^{3}$, i.e. 11 times heavier than the yarn. This makes the yarn a potential candidate to replace metallic wires in applications where weight is an important issue, such as in aerospace applications.

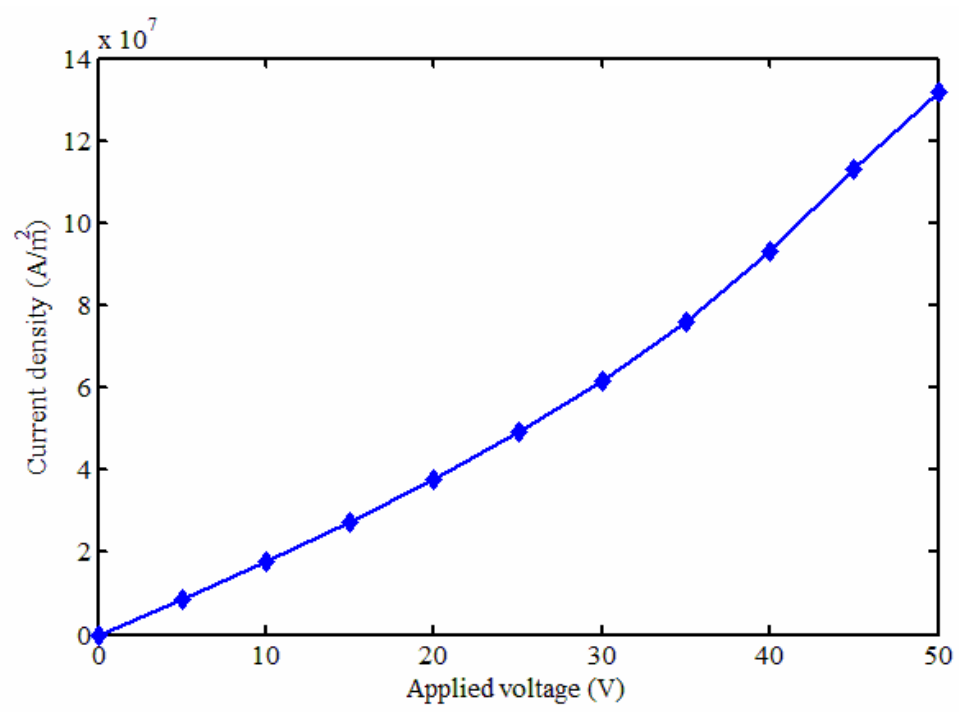

Figure 9: The maximum current density in the yarn reached before the yarn breaks.

\section{CONCLUSIONS}

Twist-spun yarns of multi-walled carbon nanotubes were shown to work as electromechanical actuators, force sensors and current carriers/microwires. Considering their very low density of $0.8 \mathrm{~g} / \mathrm{cm}^{3}$, they look quite promising for these applications. Strains of up to $0.2 \%$ at loads of up to $200 \mathrm{MPa}$ have been achieve in actuation mode, while sharp and easily detectable current spikes of up to $80 \mathrm{nA}$ are generated in force sensory mode. The yarns can carry large current densities, reaching $1.4 \times 10^{8} \frac{\mathrm{A}}{\mathrm{m}^{2}}$. This, along with their low density makes them candidates to act as current carriers even at large length scales, and especially in situations such as aerospace applications were cargo weight is a major issue.

\section{REFERENCES}

[1] Baughman, R. H., Zakhidov, A. A. and de Heer, W. A., "Carbon nanotubes - the route toward applications", Science, 297, 787-792 (2002)

[2] Zhang, M., Atkinson, K. R. and Baughman, R. H., "Multifunctional carbon nanotube yarns by downsizing an ancient technology", Science, 306, 1358-1361 (2004)

[3] Atkinson, K. R., Hawkins, S. C., Huynh, C., Skourtis, C., Dai, J., Zhang, M., Fang, S., Zakhidov, A. A., Lee, S. B., Aliev, A. E., Williams, C. D. and Baughman, R. H., "Multifunctional carbon nanotube yarns and transparent sheets: Fabrication, properties, and applications", Physica B: Condensed Matter, 394, 339-343 (2007)

[4] Mirfakhrai, T., Oh, J., Kozlov, M., Fok, E. C. W., Zhang, M., Fang, S., Baughman, R. H. and Madden, J. D. W., "Electrochemical actuation of carbon nanotube yarns", Smart Materials and Structures, 16, S243 (2007) 
[5] Wu, Y., Alici, G., Madden, J. D. W., Spinks, G. M. and Wallace, G. G., "Soft mechanical sensors through reverse actuation in polypyrrole", Advanced Functional Materials, 17, 3216-3222 (2007 )

[6] Gartstein, Y. N., Zakhidov, A. A. and Baughman, R. H., "Charge-induced anisotropic distortions of semiconducting and metallic carbon nanotubes", Physical Review Letters, 89, (2002)

[7] Mirfakhrai, T., Oh, J., Kozlov, M., Fang, S., Zhang, M., Baughman, R. H. and Madden, J. D. W., "High Strain Actuation of Carbon Nanotube Yarns in Ionic Liquids", In preparation, (In preparation)

[8] Tu, Z.-c. and Ou-Yang, Z.-c., "Single-walled and multiwalled carbon nanotubes viewed as elastic tubes with the effective Young's moduli dependent on layer number", Physical Review B, 65, 233407 (2002)

[9] Hearle, J. W. S., Grossberg, P. and Backer, S., "Structural Mechanics of Fibers, Yarns and Fabrics", WileyInterscience New York (1969)

[10] Mirfakhrai, T., Oh, J., Kozlov, M., Fang, S., Zhang, M., Baughman, R. H. and Madden, J. D. W., "Carbon nanotube yarns as mechanical sensors and generators", In preparation, (In preparation)

[11] Maruyama, R., "The electrical transport properties of C60 treated by argon plasma", Journal of Molecular Structure, 831, 10-17 (2007)

[12] Aliev, A. E., Guthy, C., Zhang, M., Fang, S., Zakhidov, A. A., Fischer, J. E. and Baughman, R. H., "Thermal transport in MWCNT sheets and yarns", Carbon, 45, 2880-2888 (2007) 